\title{
Effect of ultraviolet on vitamin D and quality of life in postmenopausal women: a randomized controlled study
}

\author{
DOI: https://doi.org/10.5114/pq.2019.84272
}

\author{
Dina Mahmoud Aleshmawy Mohamed', Fahima Metwally Okeil', Amel Mohamed Yousef', \\ Mohammed Hassan Mustafa ${ }^{2}$, Hamada Ahmed Hamada ${ }^{3}$, Ibtissam M. Saab ${ }^{4}$ \\ ${ }^{1}$ Department of Physical Therapy for Women's Health, Faculty of Physical Therapy, Cairo University, Giza, Egypt \\ ${ }^{2}$ Faculty of Medicine, Cairo University, Giza, Egypt \\ ${ }^{3}$ Department of Biomechanics, Faculty of Physical Therapy, Cairo University, Giza, Egypt \\ ${ }^{4}$ Department of Physical Therapy, Faculty of Health Sciences, Beirut Arab University, Beirut, Lebanon
}

\section{Abstract}

Introduction. To determine the influence of ultraviolet radiation on vitamin D plasma levels and quality of life in postmenopausal women with vitamin D deficiency.

Methods. In a single-blinded randomized controlled trial design, 30 postmenopausal women were chosen suffering from a progressive, generalized muscle weakness, associated with decrease in physical function and poor quality of life. The subjects were aged 45-65 years and were randomly assigned into group $A$, treated by ultraviolet radiation in addition to regular aerobic exercises; and group B, receiving solely aerobic exercises. All participants had 3 sessions per week for 3 months. Outcome measures determined before and after the 3 months of intervention were vitamin D level and the results of the short form (SF-36) of a specific quality of life questionnaire.

Results. A significant increase in vitamin D was observed along with an improvement in quality of life in group A compared with group $B$ after the treatment period.

Conclusions. The 12-week program of ultraviolet radiation in addition to regular aerobic exercises yielded more improvement in vitamin $\mathrm{D}$ and quality of life than aerobic exercises alone in the management of postmenopausal chronic musculoskeletal disorders.

Key words: vitamin D, quality of life, ultraviolet radiation, aerobic exercises

\section{Introduction}

Menopause is determined as the interruption of menstruation for more than 1 year as a result of degenerative changes in the ovaries and follicles, and is usually associated with changes in the ovarian hormone levels. The part of life after menopause is commonly termed postmenopausal period in female life [1]. The clinical picture associated with menopause is usually the presence of hot flushes, irregular menses, and excessive night sweats. While some women report no major signs other than the discontinuation of menses [2], many others tend to report numerous somatic and psychological symptoms, often designated as postmenopausal syndrome.

It is well documented that age is linked to a decline in physiological functions, particularly the loss of bone mass density (BMD) in addition to a drop in muscle strength and mass termed sarcopenia. Such decline in muscle mass has been accused to induce functional impairment, especially related to activities of daily living, in addition to increased risk of falling and nonvertebral fractures [3].

Vitamin D deficiency is often observed in postmenopausal women with parathyroid hormone suppression and is associated with increased intestinal calcium absorption, reduced $\mathrm{BMD}$, increased bone loss, and higher fracture rate. Various risk factors have been identified, including age, de- creased dietary intake, diminished sunlight exposure, wintertime, nursing home environment, reduced skin thickness, decline in the ability of the skin to produce vitamin $D$, impaired intestinal uptake, and decreased hydroxylation in both kidneys and liver [4]. Vitamin D deficiency is usually asymptomatic, but research conclusions reported generalized muscle weakness, lengthened time to peak contraction in addition to augmented time to muscle relaxation in postmenopausal women. Clinically, such associated changes are often described by affected women as heaviness in the legs, tiring easily, difficulty when performing some activities of daily living, particularly rising from a seated or squat position, mounting stairs, or lifting objects [5]. Diagnosis of vitamin D deficiency is crucial since the disorder may lead to impairment in quality of life, even if no specific symptoms are present [6].

Vitamin D supplementation is commonly used as a medical intervention but overdose may cause hypercalcemia, with associated anorexia, nausea, vomiting and diarrhoea, polyuria, polydipsia, weakness, weight loss, insomnia, irritability, nervousness, severe depression, pruritus, proteinuria, and potential calcification in the kidneys, and ultimately renal failure [7]. Non-pharmaceutical treatment, such as ultraviolet radiation and exercises, is used to address postmenopausal symptoms, especially that chronic musculoskeletal disorders have been well correlated with diminished quality of life

Correspondence address: Hamada Ahmed, Department of Biomechanics, Faculty of Physical Therapy, Cairo University, 7 Ahmed Elziat Street, Bean Elsariat, El Dokki, Giza, Cairo, Egypt, e-mail: Hamada.Ahmed@pt.cu.edu.eg 
scores. Such modalities are regarded as natural and safe in managing postmenopausal syndrome, and less invasive than medications [8]. Ultraviolet is electromagnetic energy which falls between visible rays and $\mathrm{X}$-rays and has a wavelength between 10 and $400 \mathrm{~nm}$. It is divided into UVA (wavelength range of 315-400 nm), UVB (wavelength range of 280-315 $\mathrm{nm}$ ), and UVC (wavelength below $280 \mathrm{~nm}$ ) [9]. The skin absorbs UVB radiation and uses it to convert skin sterols precursors, such as 7-dehydrocholesterol, to cholecalciferol (vitamin $\mathrm{D}_{3}$ ). In the liver and kidneys, this latter is converted to the active metabolite form $1,25(\mathrm{OH})_{2} \mathrm{D}_{3}$. It has been agreed for many years that exposure to artificial ultraviolet radiation or artificial sunlight can be curative for vitamin $D$ deficiencies [10]. In the same context, aerobic exercise has been claimed to be efficient in the management of a number of postmenopausal signs and symptoms, including hot flushes, osteoarthritis, hyperlipidaemia, fibromyalgia, depression, and diabetes mellitus [11]. Regular aerobic activities were also reported to maintain and improve quality of life, decrease fatigue sensation, and lessen the rate of depressive disorders [12].

Researches trying to figure the relation between vitamin $D$ deficiency and skeletal muscle strength concluded opposing results. Such conflicting results might be caused by research methodology differences, genetic causes, and study participants' characteristics. Additionally, vitamin D deficit has been found to be one of the correlated risk factors for osteoporosis, high fall risk, and fractures, and was accused to negatively affect the patients' quality of life. Thus, the present study aimed to investigate the effect of ultraviolet radiation and aerobic exercise on muscle strength and, consequently, the quality of life in women in their postmenopausal life period.

\section{Subjects and methods}

\section{Participants}

Overall, 36 postmenopausal females were enrolled from the women's health outpatient clinic of Obstetrics and Gynaecology Department at the Faculty of Physical Therapy, Cairo University. Eligible participants' age ranged between 45 and 56 years, and their body mass index ranged between 28 and $30 \mathrm{~kg} / \mathrm{m}^{2}$; the patients reported a decrease in their physical function and quality of life. Exclusion criteria consisted of involvement in any physical activity, even in leisure time, for the previous 12 months or suffering from any acute or chronic metabolic, hormonal, neurological, musculoskeletal, gastrointestinal, or urinary disorder or disease. In addition, the subjects included in the study did not take any vitamin D supplements, osteoporosis treatment, or current or previous hormone replacement therapy. A total of 30 women were randomized. The study was performed between September 2017 and October 2018.

\section{Intervention}

This was a prospective, single-blinded, pre- and post-test randomized controlled trial. All participants were given a brief demonstration about the study and the tasks to be performed. An independent research assistant unwrapped closed casings containing random cards generated automatically by a computer and divided the participants into 2 identical groups, A and B. The study did not record dropouts after randomization. Group A received ultraviolet radiation in addition to regular aerobic exercises. Ultraviolet sessions were performed 3 times per week for 3 months in patients lying down comfortably. The aerobic exercises were implemented
3 times per week for 3 months. Group B received solely aerobic exercises, 3 sessions per week for 3 months. For both groups, the aerobic exercise sessions involved 40 minutes of treadmill walking, as follows: warm-up (10 minutes), followed by exercising ( 20 minutes), and cooling down (10 minutes). The intensity of the exercise session was fixed at $60-70 \%$ of maximum heart rate, calculated as 220 - age [13].

\section{Outcome measures}

The plasma concentrations of vitamin $\mathrm{D}$ were recorded before and after the 3 months of intervention in both groups with the use of high-performance liquid chromatography (HPLC) (Shimadzu, Japan). The applied kits allowed the chromatographic determination of 25-OHD on a simple isocratic HPLC system with ultraviolet detection. Interfering components were removed and analyses were concentrated through efficient protein precipitation and selective solid phase extraction. The limit of detection was $2.5 \mathrm{ng} / \mathrm{ml}$ and the coefficient of variation was $<7 \%$. It has been found that serum 25-hydroxyvitamin $D$ can be considered as one of the most valid markers of vitamin $D$ status. It has been also agreed that vitamin $D$ is deficient with levels less than $20 \mathrm{ng} / \mathrm{ml}$ (50 nmol/l) of 25-hydroxyvitamin $\mathrm{D}$, while the range of $21-$ $29 \mathrm{ng} / \mathrm{ml}(52.5-72.5 \mathrm{nmol} / \mathrm{l})$ is referred to as vitamin D insufficiency, and the ideal level is set as $30 \mathrm{ng} / \mathrm{ml}(75 \mathrm{nmol} / \mathrm{l})$. In this study, a level less than $20 \mathrm{ng} / \mathrm{ml}$ of 25-hydroxyvitamin $\mathrm{D}$ was considered as marker of vitamin D deficiency [14].

Additionally, the quality of life was evaluated by the short form (SF-36) questionnaire, a tool regarded generic and multidimensional, and evaluating a person's overall satisfaction with life and health. The tool has been translated and validated into many languages, including Arabic [15]. The Arabic version of the questionnaire was self-administered and all subjects completed it at their routine clinical visit after they had accepted to participate in the study. SF-36 is a measure of physical and mental status within the previous 4 weeks, and consists of 3 levels: one containing 36 items, the second level comprising 8 sub-scales that aggregate $2-10$ items, and the third level involving 2 summary scales that aggregate sub-scales. Levels 2 and 3 aggregate 4 sub-scales each: the first addressing the physical component, and the other addressing the mental component score. General health, vitality, and social functioning have a correlation with both of the summary scales. The higher the score, the better the quality of life, with scores ranging between 0 and 100 .

\section{Data analyses}

The recorded data were analysed by using the Statistical Package for the Social Sciences (SPSS) software, version 23. Before the concluding analysis, data were evaluated for normality assumption, homogeneity, and occurrence of extreme scores. This analysis was performed as preliminary measure before parametric calculations of the difference analysis. We recorded a linear relationship between the dependent variables using a scatterplot, and no proof of multicollinearity using Pearson correlation $(|r|<0.9)$. Additionally, no univariate outliers were observed in the data through a boxplot, and no multivariate outliers as evidenced by Mahalanobis distance. Vitamin $D$ and quality of life were normally distributed, as demonstrated by the Shapiro-Wilk's test $(p>$ $0.05)$. Levene's test assured the homogeneity of variances $(p>0.05)$ for all dependent variables. Consequently, $2 \times 2$ MANOVA (mixed design) was considered to match the recorded variables for groups and at various periods. The alpha 
level was set at 0.05 . The sample size of 30 participants was calculated to yield an $85 \%$ power at $\alpha=0.05$.

\section{Ethical approval}

The research related to human use has complied with all the relevant national regulations and institutional policies, has followed the tenets of the Declaration of Helsinki, and has been approved by the authors' institutional review board at the Faculty of Physical Therapy, Cairo University with a reference number P.T.REC/012/001543.

\section{Informed consent}

Informed consent has been obtained from all individuals included in this study.

\section{Results}

A total of 30 participants were involved in the final analysis after recording drops of 6 volunteers related to personal decisions of the participants (Figure 1). They were previously allotted into 2 groups: group A (15 patients) received ultraviolet radiation in addition to regular aerobic exercises, and group B (15 patients) received aerobic exercises. The independent $t$-test showed no significant differences $(p>0.05)$ when comparing mean values of different personal characteristics in both groups (Table 1).

Mixed design MANOVA investigated 30 patients allocated into 2 groups identical in numbers. It showed a significant within-subject effect $(\mathrm{F}=124.358, p=0.0001)$ and treatment * time effect $(F=35.942, p=0.0001)$. On the other hand, there was significance in the between-subject effect $(F=12.289$, $p=0.0001$ ). Table 2 presents descriptive statistics (mean $\pm S D$ ) and multiple pairwise comparison tests for the measured variables. Additionally, regarding the within-subject effect, the multiple pairwise comparison tests uncovered a significant increase $(p<0.05)$ in vitamin $D$ levels and quality of life scores after treatment in comparison with before-treatment means for both groups. Calculations of the between-subject effects with the use of multiple pairwise comparisons revealed a more significant increase $(p<0.05)$ in both vitamin $D$ and quality of life in group $A$ than in group $B$.

\section{Discussion}

It has been found that musculoskeletal disorders account for $14-30 \%$ of disorders affecting postmenopausal women resulting in functional deterioration, increase in the requirement for support in the achievement of routine activities, and poor quality of life [16]. The main cause for such limitations is the decline in oestrogen levels, leading to progressive drops in the levels of growth hormone and insulin-like growth factor 1 . The associated decline in androgen production is accounted for the onset of sarcopenia [17]. The risk of sarcopenia in postmenopausal women has been reported to be doubled when concentrations of 25(OH)D drop lower than $10 \mathrm{ng} / \mathrm{ml}$. Vitamin D deficiency is generally silent and usually women exhibit no clear symptoms, even though some amounts of muscle weakness and pain might be present and possibly lead to further reduction in the woman's quality of life [18].

In the present study, results revealed a significant rise in vitamin $D$ levels and enhancement in the quality of life questionnaire scores in both post-intervention groups when compared to pre-intervention scores. However, significantly higher scores of vitamin $\mathrm{D}$ level and quality of life were recorded in subjects treated with ultraviolet radiation after treatment when

Table 1. Personal characteristics of patients in both groups

\begin{tabular}{|l|c|c|c|c|c|}
\hline \multirow{2}{*}{ Characteristics } & Group A & Group B & \multicolumn{2}{|c|}{ Comparison } & \multirow{2}{*}{ Significance } \\
\cline { 2 - 5 } & $\bar{x} \pm S D$ & $\bar{x} \pm S D$ & $t$-value & p-value & NS \\
\hline Age (years) & $51.66 \pm 5.21$ & $53.29 \pm 5.31$ & -0.915 & 0.367 & NS \\
\hline Body mass $(\mathrm{kg})$ & $84 \pm 14.9$ & $84.05 \pm 11.28$ & -0.013 & 0.99 & NS \\
\hline Height $(\mathrm{cm})$ & $157.22 \pm 5.05$ & $157.7 \pm 3.49$ & -0.327 & 0.746 & \\
\hline
\end{tabular}

$\bar{x}$ - mean, $S D$ - standard deviation, NS - non-significant

Table 2. Descriptive statistics and multiple pairwise comparison tests for all dependent variables in both groups in different measuring periods

\begin{tabular}{|c|c|c|c|c|}
\hline \multirow{2}{*}{ Variables } & \multicolumn{2}{|c|}{ Group A } & \multicolumn{2}{|c|}{ Group B } \\
\hline & Pre-intervention & Post-intervention & Pre-intervention & Post-intervention \\
\hline Vitamin D & $20.5 \pm 1.91$ & $32.77 \pm 5.34$ & $19.22 \pm 1.88$ & $22.08 \pm 2.55$ \\
\hline Quality of life & $44.35 \pm 4.28$ & $57.05 \pm 5.44$ & $41.68 \pm 3.99$ & $46.25 \pm 5.02$ \\
\hline \multicolumn{5}{|c|}{ Within groups (pre-intervention vs. post-intervention $p$-value) } \\
\hline & \multicolumn{2}{|c|}{ Vitamin D } & \multicolumn{2}{|c|}{ Quality of life } \\
\hline Group A & \multicolumn{2}{|c|}{$0.0001^{*}$} & \multicolumn{2}{|c|}{$0.0001^{*}$} \\
\hline Group B & \multicolumn{2}{|c|}{$0.001^{*}$} & \multicolumn{2}{|c|}{$0.0001^{*}$} \\
\hline \multicolumn{5}{|c|}{ Between groups (group A vs. group B $p$-value) } \\
\hline & \multicolumn{2}{|c|}{ Vitamin D } & \multicolumn{2}{|c|}{ Quality of life } \\
\hline Pre-intervention & \multicolumn{2}{|c|}{0.064} & \multicolumn{2}{|c|}{0.075} \\
\hline Post-intervention & \multicolumn{2}{|c|}{$0.0001^{*}$} & \multicolumn{2}{|c|}{$0.0001^{*}$} \\
\hline
\end{tabular}




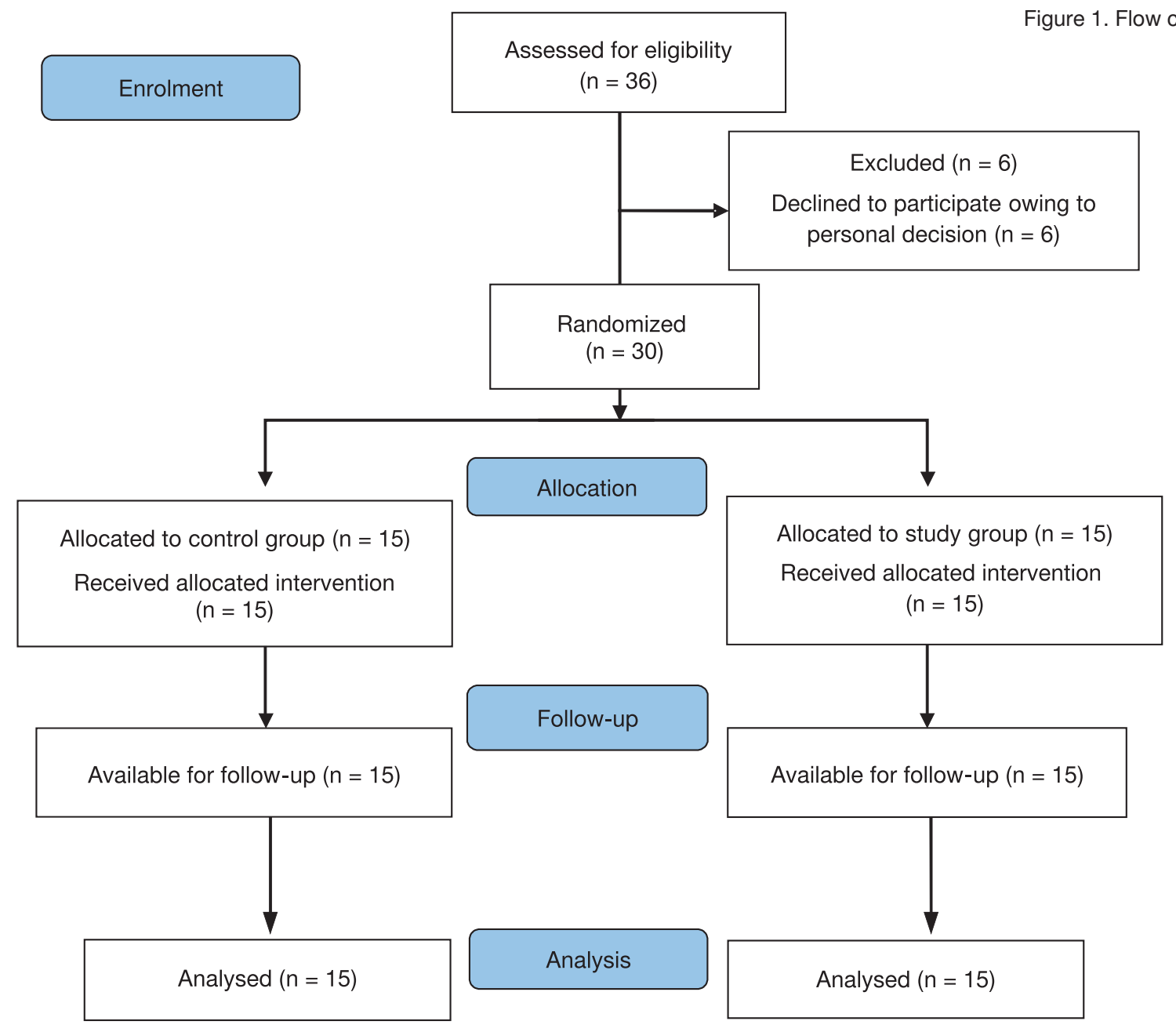

compared with controls. Such observations agree with other researchers [19], who reported that vitamin D deficiency was frequently encountered clinically in postmenopausal women and was linked to reduced intensity and duration of exposure to ultraviolet in $80-90 \%$ and to low dietary vitamin D intake in $10-20 \%$. Each of these two sources may provide sufficient supply, but if one of them is lost, the other source can easily become insufficient. In a multinational European study, the occurrence of vitamin D deficiency reached $32.1 \%$ [20], while others published higher rates [14].

In this study, remarkable improvements were recorded in the ultraviolet radiation group. This may be attributable to that the main source of vitamin $D$ is the synthesis in the skin, facilitated by the ultraviolet radiation effect on the 7-dehydrocholesterol, the vitamin D precursor. UVB has been accounted for the fabrication of vitamin $D$ in the skin, and may reach 1000 IUs for each minute of irradiation. In addition, any vitamin $\mathrm{D}$ deficiency can be caused by factors affecting the UVB photons to penetrate the skin. Dietary intake is a documented alternative source of vitamin D; on the other hand, contemporary diets lack sufficient amounts of vitamin D, even fortified diets containing high levels of dairy and/or cereal products. Thus, the human body relies highly on skin production of vitamin D; otherwise, deficiency is inevitable [21]. The observed increase in vitamin $D$ in this study is supported by other authors [22], who revealed increasing vitamin D levels even 24 hours after ultraviolet light exposure.

In the same context, the conclusions of the present study regarding improvement observed in the quality of life are in agreement with observations by other researchers [16], who studied the added value of vitamin D on regular physical ac- tivity among 77 sarcopenic postmenopausal women. Adding vitamin D supplements to gentle exercise was found useful in addressing catabolic mediators, inflammatory mediators such as C-reactive protein, and consequently refining some anabolic markers and thus increased the production of muscle proteins. These associated changes led to increase in fat-free mass and muscle strength, demonstrating effectiveness in the management of sarcopenia with additional enhancements in functional markers and quality of life.

The improvements recorded in the present study can be accounted for possible subsequent genomic and nongenomic actions leading to adequate muscle function. Genomic cascade is facilitated by nuclear vitamin $D$ receptors that influence transport of calcium in the muscles and other related metabolisms [23]. Normal serum calcium level, enabled by suitable vitamin $\mathrm{D}$ levels, can be regarded as crucial for ordinary muscle functioning. Nongenomic actions were found to take place through vitamin $D$ receptors existing on cell membranes. This binding triggers a series of pathways that were found to convey signals to the cytoplasm. Such mechanisms are also believed to regulate calcium inside muscle cells [24]. Another possible explanation could be the initiation of mitogenactivated protein kinase signalling paths that were highly correlated with myogenesis regulation, cell proliferation, differentiation, and apoptosis. Taking these mechanisms altogether, it can be deduced that vitamin D can produce stimulation of muscle cell proliferation and growth. Additionally, potential protein synthesis observed can be the result of vitamin D binding protein that is linked to adenosine triphosphate uptake in the skeletal muscle reticulum [25]. A total of 4 doses of vitamin D supplementation were found effective in enhanc- 
ing physical and mental components of SF-36 [26]. In another study [27], community dwelling women were evaluated for physical performance according to vitamin D status; the conclusion was that serum vitamin D was significantly associated with muscle strength and physical function scores recorded by SF-36 in postmenopausal women. These findings were confirmed in other studies performed in elderly women [28].

Aerobic exercises exhibited a significant improvement in quality of life. This is attributable to that regular physical activity is safe for postmenopausal women and the risks of developing major systemic or musculoskeletal disorders are reduced by regular physical activity with various identified modes and intensities [29]. The results are in line with those of other researchers [30], who recommended that postmenopausal women could benefit from the positive effects of physical activity through performing modest but regular exercise. However, a combination of various modes of training, particularly resistance and endurance training, has been claimed to be more beneficial than using one mode of exercising individually. Such variation in the training modes is claimed to contribute to improved quality of life among postmenopausal women with moderate deficits or frailty.

\section{Limitations}

Possible limitations in this study might be the psychological, physiological, and potential cultural issues.

\section{Conclusions}

In postmenopausal women, the 12-week program of ultraviolet radiation in addition to aerobic exercises yielded a significant improvement in vitamin $\mathrm{D}$ levels, and raised the score of SF-36 quality of life questionnaire when compared with aerobic exercises only.

\section{Acknowledgements}

The authors would like to acknowledge all participants involved in this study.

\section{Disclosure statement}

No author has any financial interest or received any financial benefit from this research.

\section{Conflict of interest}

The authors state no conflict of interest.

\section{References}

1. Chattha R, Raghuram N, Venkatram $P$, Hongasandra NR. Treating the climacteric symptoms in Indian women with an integrated approach to yoga therapy: a randomized control study. Menopause. 2008;15(5):862-870; doi: 10.1097/gme.0b013e318167b902.

2. Borysenko J. A woman's book of life: the biology, psychology, and spirituality of the feminine life cycle. New York: Riverhead Books; 1996.

3. Nowson CA, McGrath JJ, Ebeling PR, Haikerwal A, Daly RM, Sanders KM, et al. Vitamin D and health in adults in Australia and New Zealand: a position statement. Med J Aust. 2012;196(11):686-687; doi: 10.5694/ mja11.10301.

4. Beaudart C, Buckinx F, Rabenda V, Gillain S, Cavalier E, Slomian J, et al. The effects of vitamin D on skeletal muscle strength, muscle mass, and muscle power: a system- atic review and meta-analysis of randomized controlled trials. J Clin Endocrinol Metab. 2014;99(11):4336-4345; doi: 10.1210/jc.2014-1742.

5. Christensen MH, Apalset EM, Nordbø Y, Varhaug JE, Mellgren G, Lien EA. 1,25-dihydroxyvitamin D and the vitamin $D$ receptor gene polymorphism Apa1 influence bone mineral density in primary hyperparathyroidism. PLoS One. 2013;8(2):e56019; doi: 10.1371/journal. pone.0056019.

6. Anand S, Kaysen GA, Chertow GM, Johansen KL, Grimes B, Dalrymple LS, et al. Vitamin D deficiency, selfreported physical activity and health-related quality of life: the Comprehensive Dialysis Study. Nephrol Dial Transplant. 2011;26(11):3683-3688; doi: 10.1093/ndt/ gfr098.

7. Shaffer JA, Edmondson D, Wasson LT, Falzon L, Homma K, Ezeokoli N, et al. Vitamin D supplementation for depressive symptoms: a systematic review and metaanalysis of randomized controlled trials. Psychosom Med. 2014;76(3):190-196; doi: 10.1097/PSY.000000000000 0044.

8. Nedrow A, Miller J, Walker M, Nygren P, Huffman LH, Nelson HD. Complementary and alternative therapies for the management of menopause-related symptoms: a systematic evidence review. Arch Intern Med. 2006; 166(14):1453-1465; doi: 10.1001/archinte.166.14.1453.

9. Burnett ME, Wang SQ. Current sunscreen controversies: a critical review. Photodermatol Photoimmunol Photomed.2011;27(2):58-67;doi:10.1111/j.1600-0781.2011. 00557.x.

10. DeLuca HF. History of the discovery of vitamin D and its active metabolites. Bonekey Rep. 2014;3:479; doi: 10.1038/bonekey.2013.213.

11. Conn VS, Hafdahl AR, Porock DC, McDaniel R, Nielsen PJ. A meta-analysis of exercise interventions among people treated for cancer. Support Care Cancer. 2006; 14(7):699-712; doi: 10.1007/s00520-005-0905-5.

12. Schmitz KH, Holtzman J, Courneya KS, Mâsse LC, Duval S, Kane R. Controlled physical activity trials in cancer survivors: a systematic review and meta-analysis. Cancer Epidemiol Biomarkers Prev. 2005;14(7):15881595; doi: 10.1158/1055-9965.EPI-04-0703.

13. Chagas EFB, Bonfim MR, Turi BC, Brondino NCM, Monteiro HL. Effect of moderate-intensity exercise on inflammatory markers among postmenopausal women. J Phys Act Health. 2017;14(6):479-485; doi: 10.1123/jpah.20160319.

14. Quesada-Gómez JM, Diaz-Curiel M, Sosa-Henriquez M, Malouf-Sierra J, Nogues-Solan X, Gomez-Alonso C, et al. Low calcium intake and inadequate vitamin $D$ status in postmenopausal osteoporotic women. J Steroid Biochem Mol Biol. 2013;136:175-177; doi: 10.1016/j.jsbmb.2012. 10.013 .

15. Guermazi M, Allouch C, Yahia M, Huissa TB, Ghorbel S, Damak J, et al. Translation in Arabic, adaptation and validation of the SF-36 Health Survey for use in Tunisia. Ann Phys Rehabil Med. 2012;55(6):388-403; doi: 10.1016/j. rehab.2012.05.003.

16. Rondanelli M, Klersy C, Terracol G, Talluri J, Maugeri R, Guido D, et al. Whey protein, amino acids, and vitamin D supplementation with physical activity increases fat-free mass and strength, functionality, and quality of life and decreases inflammation in sarcopenic elderly. Am J Clin Nutr. 2016;103(3):830-840; doi: 10.3945/ajcn.115.113357.

17. Lowe DA, Baltgalvis KA, Greising SM. Mechanisms behind estrogen's beneficial effect on muscle strength in 
females. Exerc Sport Sci Rev. 2010;38(2):61-67; doi: 10.1097/JES.0b013e3181d496bc.

18. Basaran S, Guzel R, Coskun-Benlidayi I, Guler-Uysal F. Vitamin D status: effects on quality of life in osteoporosis among Turkish women. Qual Life Res. 2007;16(9):14911499; doi: 10.1007/s11136-007-9257-6.

19. Civelek GM, Pekyavas NO, Cetin N, Cosar SN, Karatas M. Association of vitamin $\mathrm{D}$ deficiency with muscle strength and quality of life in postmenopausal women. Climacteric. 2014;17(4):472-477; doi: 10.3109/13697137.2014. 898265.

20. Bruyère $O$, Malaise $O$, Neuprez $A$, Collette J, Reginster JY. Prevalence of vitamin D inadequacy in European postmenopausal women. Curr Med Res Opin. 2007;23(8): 1939-1944; doi: 10.1185/030079907X219562.

21. Cicarma E, Mørk C, Porojnicu AC, Juzeniene A, Tam TT, Dahlback A, et al. Influence of narrowband UVB phototherapy on vitamin $D$ and folate status. Exp Dermatol. 2010;19(8):e67-e72; doi: 10.1111/j.1600-0625.2009. 00987.x.

22. Wortsman J, Matsuoka LY, Chen TC, Lu Z, Holick MF. Decreased bioavailability of vitamin D in obesity. Am J Clin Nutr. 2000;72(3):690-693; doi: 10.1093/ajcn/72.3.690.

23. Bartoszewska M, Kamboj M, Patel DR. Vitamin D, muscle function, and exercise performance. Pediatr Clin North Am. 2010;57(3):849-861; doi: 10.1016/j.pcl.2010.03.008.

24. Ceglia L. Vitamin D and its role in skeletal muscle. Curr Opin Clin Nutr Metab Care. 2009;12(6):628-633; doi: 10.1097/MCO.0b013e328331c707.

25. Philippou A, Maridaki M, Halapas A, Koutsilieris M. The role of the insulin-like growth factor 1 (IGF-1) in skeletal muscle physiology. In Vivo. 2007;21(1):45-54.

26. Sakalli $H$, Arslan D, Yucel AE. The effect of oral and parenteral vitamin D supplementation in the elderly: a prospective, double-blinded, randomized, placebocontrolled study. Rheumatol Int. 2012;32(8):22792283; doi: 10.1007/s00296-011-1943-6.

27. Zamboni M, Zoico E, Tosoni P, Zivelonghi A, Bortolani A, Maggi $S$, et al. Relation between vitamin D, physical performance, and disability in elderly persons. J Gerontol A Biol Sci Med Sci. 2002;57(1):M7-M11; doi: 10.1093/ gerona/57.1.m7.

28. Houston DK, Cesari M, Ferrucci L, Cherubini A, Maggio $D$, Bartali $B$, et al. Association between vitamin $D$ status and physical performance: the InCHIANTI study. J Gerontol A Biol Sci Med Sci. 2007;62(4):440-446; doi: 10.1093/gerona/62.4.440.

29. Davidson LE, Hudson R, Kilpatrick K, Kuk JL, McMillan K, Janiszewski PM, et al. Effects of exercise modality on insulin resistance and functional limitation in older adults: a randomized controlled trial. Arch Intern Med. 2009; 169(2):122-131; doi:10.1001/archinternmed.2008.558.

30. Weening-Dijksterhuis E, de Greef MH, Scherder EJ, Slaets JP, van der Schans CP. Frail institutionalized older persons: a comprehensive review on physical exercise, physical fitness, activities of daily living, and quality-oflife. Am J Phys Med Rehabil. 2011;90(2):156-168; doi: 10.1097/PHM.0b013e3181f703ef. 